\title{
Modeling the Potential Distribution of Machilus thunbergii under the Climate Change Patterns in China
}

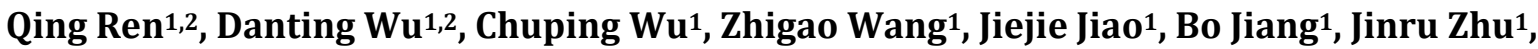 \\ Yujie Huang1, Tingting Li ${ }^{1}$, Weigao Yuan ${ }^{*}$
}

${ }^{1}$ Zhejiang Academy of Forestry, Hangzhou, China

${ }^{2}$ School of Forestry and Biotechnology, Zhejiang Agriculture and Forestry University, Hangzhou, China

Email: *zfaywg@126.com

How to cite this paper: Ren, Q., Wu, D. T., Wu, C. P., Wang, Z. G., Jiao, J. J., Jiang, B., Zhu, J. R., Huang, Y. J., Li, T. T., \& Yuan, W. G. (2020). Modeling the Potential Distribution of Machilus thunbergii under the Climate Change Patterns in China. Open Journal of Forestry, 10, 217-231.

https://doi.org/10.4236/ojf.2020.102015

Received: January 13, 2020

Accepted: March 6, 2020

Published: March 9, 2020

Copyright (c) 2020 by author(s) and Scientific Research Publishing Inc. This work is licensed under the Creative Commons Attribution International License (CC BY 4.0).

http://creativecommons.org/licenses/by/4.0/

\begin{abstract}
The potential geographic distribution and favorable climatic conditions of Machilus thunbergii under current and future predicted climates in China are predicted based on MaxEnt model and ArcGIS software. The results show that the AUC values in different time periods and emission seniors are more than 0.9 , which indicates the prediction is excellent. Precipitation of the coldest quarter, precipitation of the driest month, annual precipitation, mean diurnal range, and temperature annual range are the most important environmental factors affecting the distribution of Machilus thunbergii. At present, the suitable areas of Machilus thunbergii are mainly concentrated in the eastern subtropics of China, with a total area of $118.47 \times 10^{4} \mathrm{~km}^{2}$. The medium-suitability area and the high-suitability area are concentrated in Wuyi Mountains, Luoxiao Mountains, Xuefeng Mountains, Nanling and east of Taiwan Mountains. With the change of climate, the suitable area increases, and the medium-suitability area and high-suitability area migrate and expand to the east, and the low-suitability area expands slightly to the west and north. So, the simulated distribution of Machilus thunbergii should be one of priorities, when instigating in-situ conservation. The research results can provide a theoretical reference for the popularization and planting of Machilus thunbergii.
\end{abstract}

\section{Keywords}

Machilus thunbergii, Species Distribution, Climate Change, Environmental Factors 


\section{Introduction}

Due to the rapid urbanization and industrialization, a great number of forests were cut to meet the market demand for wood. The global forest area decreased from 4.28 billion hectares to 3.99 billion hectares in 1990-2015, and the forest cover rate decreased from $31.85 \%$ to $30.85 \%$ (FAO, 2015), leading to the sharp deterioration of the global ecological environment, the continuous decline of natural forest resources, and many plants became endangered species. Significant progress has been made in increasing permanent forests through natural regeneration and artificial afforestation, with the area of plantations being expected to reach 300 million hectares by 2020 (FAO, 2010). However, the status of plantation development shows that there are few large diameter timber trees and precious timber species, and the structural contradiction between supply and demand of high-quality timber is very prominent (SFA, 2014). Therefore, studying the spatial distribution and predicting the potential distribution area under the climate change is of great significance for guiding the natural regeneration and constructing plantation.

Machilus thunbergii (Machilus Nees, Lauraceae), is an evergreen broad-leaved tree preferring warm and humid climate. It has slightly shade tolerant, and is mostly found in the mountain broadleaved forest below $800 \mathrm{~m}$, and distributes in subtropical and warm temperate zone of China (Jiang \& Yu, 2001; Wu et al., 2008). Machilus thunbergii is also a fine timber tree with great artificial cultivation value and development potential, and is widely used in gardening, timber, spice extraction and pharmaceutical preparation (Hu, 2002; Ma \& Huang, 2019; Zhang et al., 2019). Due to the destruction of natural resources and ecological environment, Machilus thunbergii has been listed as a third-class national key protected wild plant in China. However, previous research on Machilus thunbergii mainly focuses on the community structure and photosynthetic physiological characteristics (Wu et al., 2006; Zhang, 2010; Hwang et al., 2011; Kaneko et al., 2012) without a definite division of its suitable distribution. The research on the suitable distribution and environmental adaptability of Machilus thunbergii in China under the climate change is still in blank.

The maximum entropy modeling (MaxEnt) is widely used in the prediction of potential distribution of species in the context of climate change, environmental factor detection and risk assessment of invasive species (Adhikari et al., 2012; Yang et al., 2013; Fandohan et al., 2015; Sung et al., 2018; Chen et al., 2019), based on geographical distribution data and a variety of environmental data. Compared with other models, the MaxEnt has the advantages of less sample size, less influence by sample deviation and better accuracy (Elith et al., 2011; Kramer-Schadt et al., 2013).

Therefore, studying the spatial distribution and predicting the potential distribution area of Machilus thunbergii under the climate change is of great significance for determining appropriate biodiversity conservation and strategies for the recovery of Machilus thunbergii resources. 


\section{Materials and Methods}

\subsection{Data Collection}

The geographical distribution data of Machilus thunbergii is derived from China Digital Herbarium (CVH, http://www.cvh.ac.cn/), China National Specimen Resource Platform (NSII, http://www.nsii.org.cn/) and the Global Biodiversity Information Network (GBIF, https://www.gbif.org/). After screening and eliminating some missing and duplicated data, a total of 277 effective distribution points (Figure 1) were obtained in China.

Climate data are derived from the World Meteorological Database (Worldclim, http://www.worldclim.org/) with a spatial resolution of 2.5 ' (about $22 \mathrm{~km}^{2}$ ). The 19 environmental variable contains comprehensive data of temperature and precipitation, which are the most influential factors for the plant distribution (Fick \& Hijmans, 2017). And the 19 environmental variables (Table 1) data were obtained based on ArcGIS 10.0. Select the climate data from1950 to 2000 as the current climate, which matches the data of the specimen bank. And according to the classification of future climate by the World Meteorological Database, select the climate data of 2050s (2041-2060) and 2070s (2061-2080) as the future climate data.

Based on a CCSM system model with a high degree of fitness in China's climatic simulation (Tian \& Jiang, 2013), the emission scenario (The greenhouse gas concentrations and their extensions from 1765 to 2300) selects RCP4.5 (Stable $\mathrm{CO}_{2}$ emission scenario), RCP8.5 (the highest $\mathrm{CO}_{2}$ emission scenario), representing $\mathrm{CO}_{2}$ emission scenario of rising GHG concentrations in the future (Le Quéré et al., 2018; Fick \& Hijmans, 2017).

\subsection{Statistical Analysis}

The geographical distribution data of Machilus thunbergii and 19 variables are imported into Maxent. In order to ensure higher reliability of result analysis,

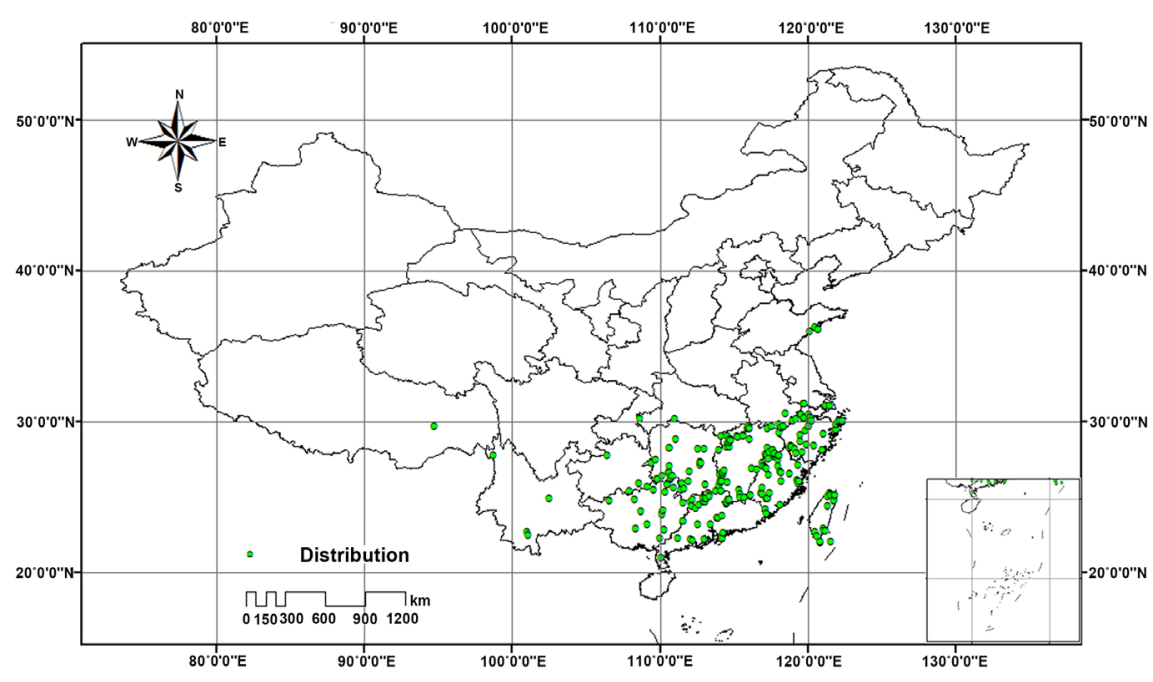

Figure 1. Geographic distributions of Machilus thunbergii. 
Table 1. 19 environmental variables used in study.

\begin{tabular}{|c|c|c|c|c|c|}
\hline Code & Environment variable & Unit & Code & Environment variable & Unit \\
\hline Biol & Annual mean temperature & ${ }^{\circ} \mathrm{C}$ & Biol1 & Mean temperature of coldest quarter & ${ }^{\circ} \mathrm{C}$ \\
\hline $\mathrm{Bio} 2$ & Mean diurnal range & ${ }^{\circ} \mathrm{C}$ & Biol2 & Annual precipitation & $\mathrm{mm}$ \\
\hline Bio3 & Isothermality & - & Biol3 & Precipitation of wettest month & $\mathrm{mm}$ \\
\hline Bio4 & Temperature seasonality & - & Biol4 & Precipitation of driest month & $\mathrm{mm}$ \\
\hline Bio5 & Maximum temperature of warmest month & ${ }^{\circ} \mathrm{C}$ & Bio15 & Precipitation seasonality & - \\
\hline Bio6 & Minimum temperature of coldest month & ${ }^{\circ} \mathrm{C}$ & Biol6 & Precipitation of wettest quarter & $\mathrm{mm}$ \\
\hline Bio7 & Temperature annual range & ${ }^{\circ} \mathrm{C}$ & Biol7 & Precipitation of driest quarter & $\mathrm{mm}$ \\
\hline Bio8 & Mean temperature of wettest quarter & ${ }^{\circ} \mathrm{C}$ & Biol8 & Precipitation of warmest quarter & $\mathrm{mm}$ \\
\hline Bio9 & Mean temperature of driest quarter & ${ }^{\circ} \mathrm{C}$ & Bio19 & Precipitation of coldest quarter & $\mathrm{mm}$ \\
\hline Biol0 & Mean temperature of warmest quarter & ${ }^{\circ} \mathrm{C}$ & - & - & - \\
\hline
\end{tabular}

repeating the operation for 10 times, and selecting $75 \%$ distribution points as training data, $25 \%$ as test data. Evaluating each environmental factor by the contribution rate and the replacement important value to determine the dominant from the factor jackknife test (Elith et al., 2011; Kramer-Schadt et al., 2013).

AUC referred to the area enclosed by the ROC (receiver operation characteristic) curve and the $\mathrm{x}$-coordinate. ROC curve analysis method has been widely used in the evaluation of the potential distribution prediction model of species. It was a diagnostic test evaluation index with high recognition at present. AUC > 0.7 indicates that the prediction result is poor, AUC $>0.8$ indicates that the prediction result is general, and AUC $>0.9$ indicates that the prediction result has higher accuracy (Zhang et al., 2015).

Converting the output of the model into raster data, and calculating the area of the suitable area in ArcGIS10.0. The value of the MaxEnt output is between $0-1$, and the closer the value is to 1 , the higher the probability of species existence. According to the average spacing method (Li et al., 2018), the suitability (S) is divided into four levels: the unsuitable area $(\mathrm{S} \leq 0.2)$, the low-suitability area $(0.2<\mathrm{S} \leq 0.4)$, the medium-suitability area $(0.4<\mathrm{S} \leq 0.6)$, the high-suitability area $(\mathrm{S}>0.6)$.

\section{Results and Analysis}

\subsection{MaxEnt Model Evaluation}

According to the ROC curve output (Table 2), the average AUC in different climatic scenarios are all above 0.9 , and the accuracy of the prediction is excellent. It indicates a high fitting degree between the distribution area and the actual distribution area under different climatic scenarios. And the geographical distribution of Machilus thunbergii is nonrandom, which means environmental factors have important influence on its distribution. 
Table 2. ROC curves under different climatic conditions.

\begin{tabular}{|c|c|c|c|c|c|}
\hline \multirow{2}{*}{ AUC } & \multirow{2}{*}{$\begin{array}{c}\text { Current } \\
(1950-2000)\end{array}$} & \multicolumn{2}{|c|}{$\begin{array}{c}2050 \mathrm{~s} \\
(2041-2060)\end{array}$} & \multicolumn{2}{|c|}{$\begin{array}{c}2070 \mathrm{~s} \\
(2061-2080)\end{array}$} \\
\hline & & RCP4.5 & RCP8.5 & RCP4.5 & RCP8.5 \\
\hline Training gain & 0.965 & 0.964 & 0.961 & 0.963 & 0.960 \\
\hline Test gain & 0.958 & 0.959 & 0.958 & 0.963 & 0.953 \\
\hline
\end{tabular}

\subsection{Analysis of Contemporary Simulation Prediction Results}

\subsubsection{Main Environmental Variables Affecting the Distribution of Machilus thunbergii}

Under the current climate (Table 3), the most important environmental factor affecting the distribution was the precipitation of coldest quarter (contributing $32.2 \%$ ), followed by the annual precipitation (contributing 24.7\%), the Precipitation of driest month (contributing 15\%), the mean diurnal range (contributing 8.7\%). When referring to single variable, the Precipitation of driest month, the precipitation of coldest quarter, the Precipitation of driest quarter, the annual precipitation, and the mean diurnal range have the highest training gain, test gain and AUC value, indicating that these environmental variables are of high importance and had good compatibility. When referring to multiple variables, the temperature annual range has the smallest reduction of the highest training gain. It can be concluded that the precipitation of coldest quarter, the Precipitation of driest month, the annual precipitation, the mean diurnal range, the temperature annual range are the major climatic factors determining the distribution of Machilus thunbergii, and the sum of contribution rate these environmental factors reached $71.9 \%$.

In order to definite the effects of the major environmental factors on the suitable distribution, the curves show how the logistic prediction changes as each environmental variable, keeping all other environmental variables at their average sample value (Figure 2). The results showed that the existence probability of Machilus thunbergii was dynamically changed with the index of each environmental factor. The existence probability increased sharply while the rainfall precipitation of coldest quarter ranges from 0 to $200 \mathrm{~mm}$, the increase tends to be slow from 200 to $800 \mathrm{~mm}$, and then tends to be stable. When the precipitation of the driest month is more than $45 \mathrm{~mm}$, the existence probability increased sharply, and the range of the highest suitability is between $50-200 \mathrm{~mm}$. The existence probability increased sharply when the annual precipitation is more than 1500 $\mathrm{mm}$, and then tends to be stable between $1500-3500 \mathrm{~mm}$. When the mean diurnal range is between $3^{\circ} \mathrm{C}$ and $5^{\circ} \mathrm{C}$ and the annual precipitation is between $7^{\circ} \mathrm{C}$ and $13^{\circ} \mathrm{C}$, it meets the highest suitability for Machilus thunbergii, and the existence probability shows a sharp drop after that.

\subsubsection{Prediction of Geographical Distribution of Contemporary Machilus thunbergii}

According to the prediction results (Figure 3), the simulated suitable area (S > 0.2 ) mainly distributed at $18^{\circ} \mathrm{N}-32^{\circ} \mathrm{N}, 107^{\circ} \mathrm{E}-123^{\circ} \mathrm{E}$, in the southeast of China, 
Q. Ren et al.

Table 3. Importance analysis of environmental factors by Jackknife method.

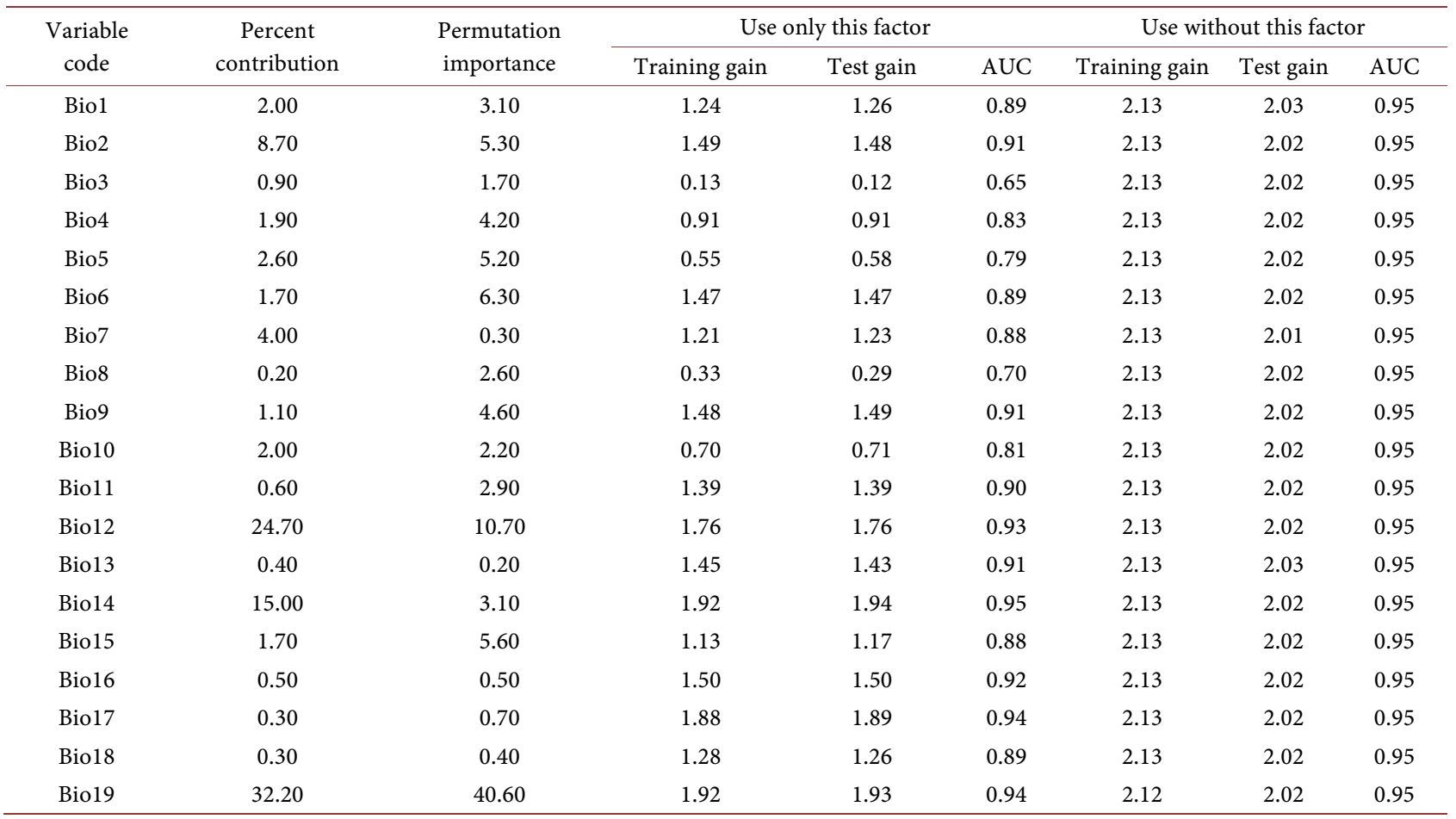

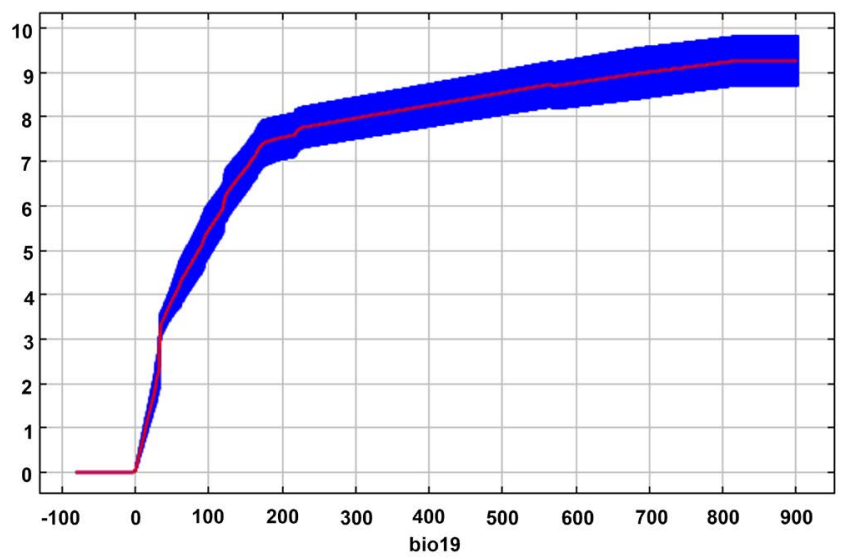

(a)

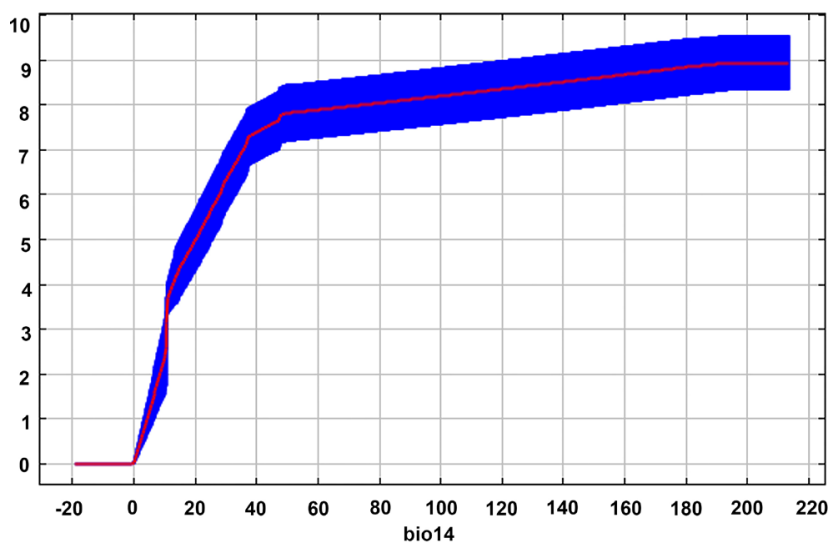

(b)

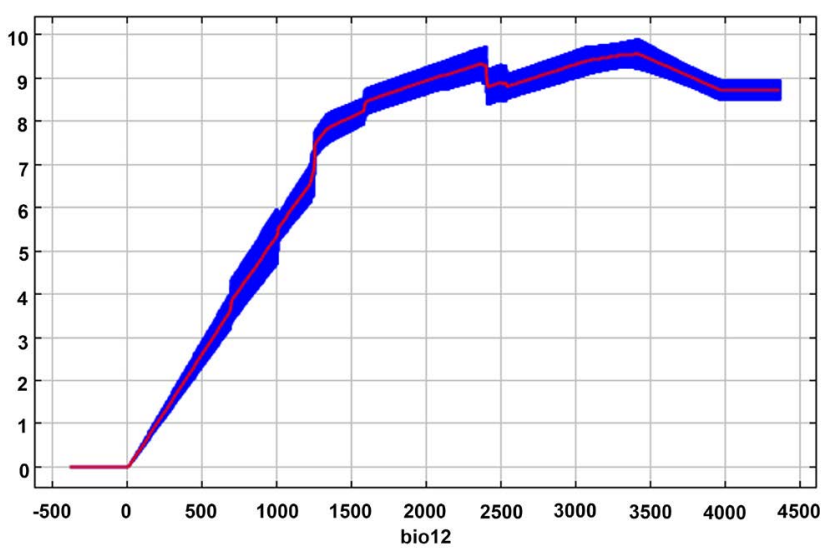

(c) 


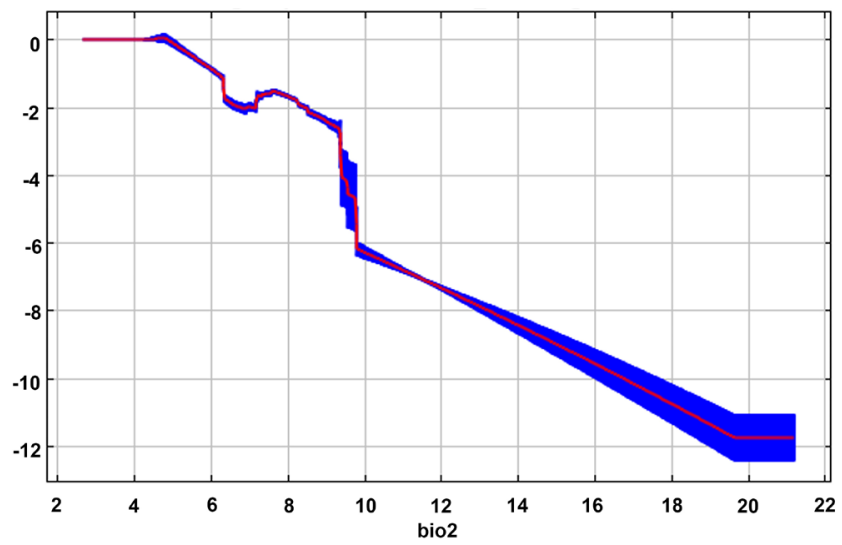

(d)

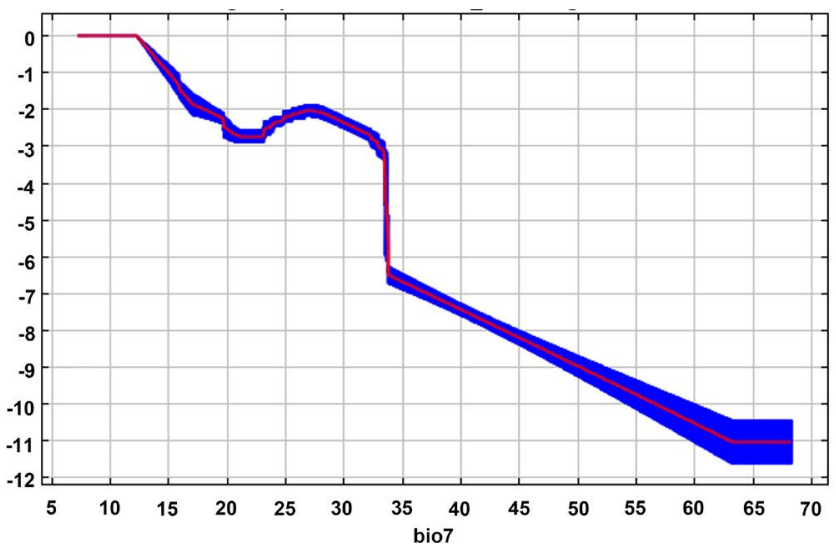

(e)

Figure 2. Response curves of major environmental factors. (a) Curve of the precipitation of coldest quarter (bio19); (b) Curve of the Precipitation of driest month (bio14); (c) Curve of the annual precipitation (bio12); (d) Curve of the mean diurnal range (bio2); (e) Curve of the temperature annual range (bio7).

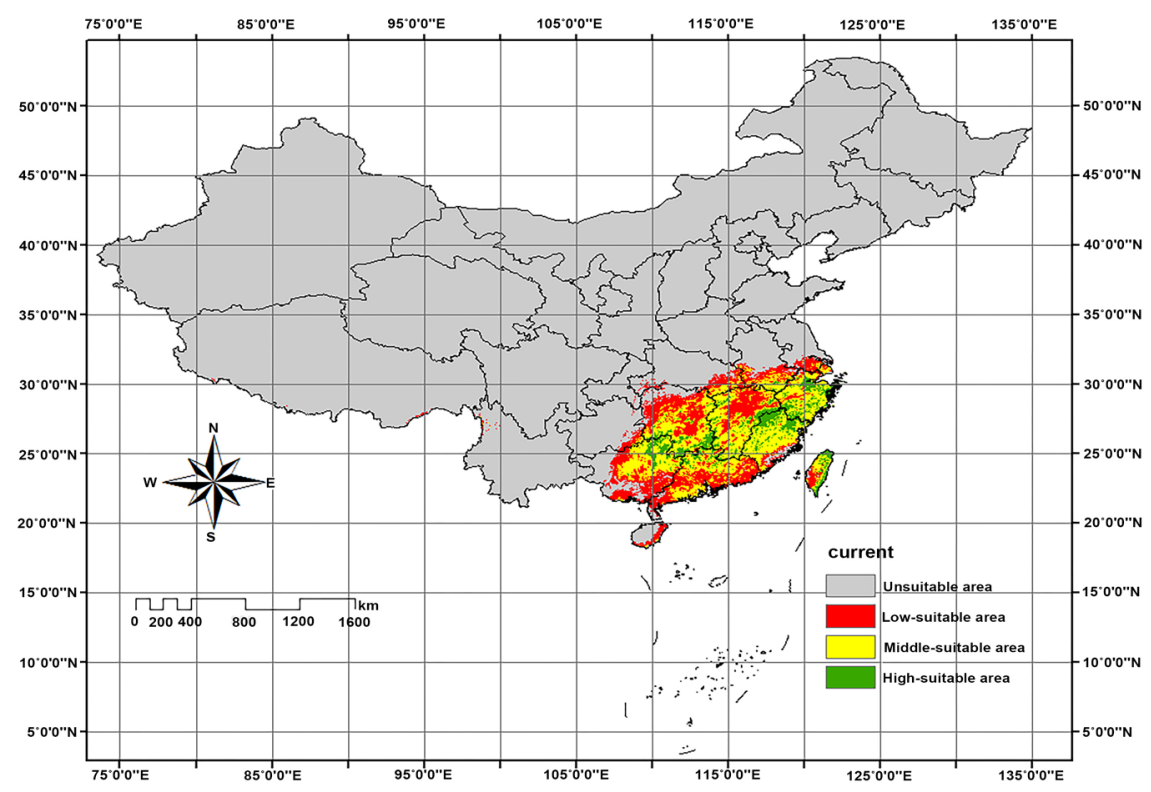

Figure 3. Distributions of Machilus thunbergii under current climatic conditions.

including Zhejiang, Fujian, Jiangxi, Hunan, Guangdong, eastern Taiwan, eastern Guangxi and southern Anhui. The total suitable area is $118.47 \times 10^{4} \mathrm{~km}^{2}$, with the areas of low-suitability area, medium-suitability area and high-suitability area account for $39.83 \%, 45.6 \%, 14.57 \%$ respectively. Under the current climatic, the distribution of suitable area of different levels is basically continuous, the medium-suitability area and high-suitability area are concentrated in Wuyi mountains, Luoxiao Mountains, Xuefeng Mountains, Nanling, and the east and north of the Taiwan Mountains.

\subsection{Potential Distribution under Different Climate Scenarios in the 2050s, 2070s}

The simulated suitable areas of Machilus thunbergii under two scenarios of 
greenhouse gas emission (RCP4.5 and RCP8.5) in the 2050s (2041-2060 years) and the 2070s (2061-2080 years), show that the distribution area is basically continuous, and gradually gathers and distributes. Compared with the current, the total suitable areas in the future increases of different degrees (Table 4, Figure $4)$.

Under the RCP4.5, the high-suitability areas tend to expand to the northeast, and gather in southern Anhui, western Zhejiang and northern Fujian, with decentralized distribution in some other areas such as Guangxi and Hunan. The medium-suitability and low-suitability areas move northward, and the fitness decreased in Guangxi and Guangdong. In the 2050s, the total suitable area of Machilus thunbergii increased by $1.33 \times 10^{4} \mathrm{~km}^{2}$, in which the high-suitability area and the low-suitability area increased by $2.33 \times 10^{4} \mathrm{~km}^{2}$, and $5.11 \times 10^{4} \mathrm{~km}^{2}$, respectively, while the medium-suitability area decreased by $6.11 \times 10^{4} \mathrm{~km}^{2}$. Compared with the 2050s, the total suitable area increased by $1.09 \times 10^{4} \mathrm{~km}^{2}$, in the 2070s, with the high-suitability and low-suitability areas increased by $2.13 \times$ $10^{4} \mathrm{~km}^{2}$, and $0.54 \times 10^{4} \mathrm{~km}^{2}$, respectively, and the medium-suitability areas decreased by $1.58 \times 10^{4} \mathrm{~km}^{2}$. Overall, the areas of high-suitability and low-suitability showed an upward trend, and the increase of low-suitability area was greater than that of high-suitability area.

Under the RCP8.5, the medium-suitability and high-suitability areas migrated to the northeast in China, concentrated in Zhejiang, Fujian, northern Guangdong, southern Anhui and northeastern Taiwan. Compared with the current, the suitability of areas in Guangxi will decline significantly. The low-suitability areas expand in Guangxi, Hunan, western Guizhou and southern Jiangsu, but receded in northern Jiangxi. In the 2050s, the total suitable area of Machilus thunbergii increased by $2.3 \times 10^{4} \mathrm{~km}^{2}$, in which the high-suitability area and the low-suitability area increased by $3.96 \times 10^{4} \mathrm{~km}^{2}$, and $5.16 \times 10^{4} \mathrm{~km}^{2}$, respectively, while the medium-suitability area decreased by $6.82 \times 10^{4} \mathrm{~km}^{2}$. Compared with the $2050 \mathrm{~s}$, the total suitable area increased by $10.87 \times 10^{4} \mathrm{~km}^{2}$, in the $2070 \mathrm{~s}$, with the low-suitability areas increased by $14.5 \times 10^{4} \mathrm{~km}^{2}$, and the medium-suitability

Table 4. Predicted suitable areas for Machilus thunbergii under different climatic conditions.

\begin{tabular}{cccccc}
\hline Decade & $\begin{array}{c}\text { Climate } \\
\text { scenarios }\end{array}$ & $\begin{array}{c}\text { Low-suitable } \\
\text { area }\left(10^{4} \mathrm{~km}^{2}\right)\end{array}$ & $\begin{array}{c}\text { Middle-suitable } \\
\text { area }\left(10^{4} \mathrm{~km}^{2}\right)\end{array}$ & $\begin{array}{c}\text { High-suitable } \\
\text { area }\left(10^{4} \mathrm{~km}^{2}\right)\end{array}$ & $\begin{array}{c}\text { Total Area } \\
\left(10^{4} \mathrm{~km}^{2}\right)\end{array}$ \\
\hline $\begin{array}{c}\text { Current } \\
(1950-2000)\end{array}$ & - & 47.19 & 54.02 & 17.26 & 118.47 \\
$\begin{array}{c}2050 \mathrm{~s} \\
(2041-2060)\end{array}$ & RCP4.5 & 52.30 & 47.91 & 19.59 & 119.80 \\
& RCP8.5 & 52.35 & 47.20 & 21.22 & 120.77 \\
$\begin{array}{c}2070 \mathrm{~s} \\
(2061-2080)\end{array}$ & RCP4.5 & 52.84 & 46.33 & 21.72 & 120.89 \\
& RCP8.5 & 66.85 & 44.27 & 20.52 & 131.64 \\
\hline
\end{tabular}




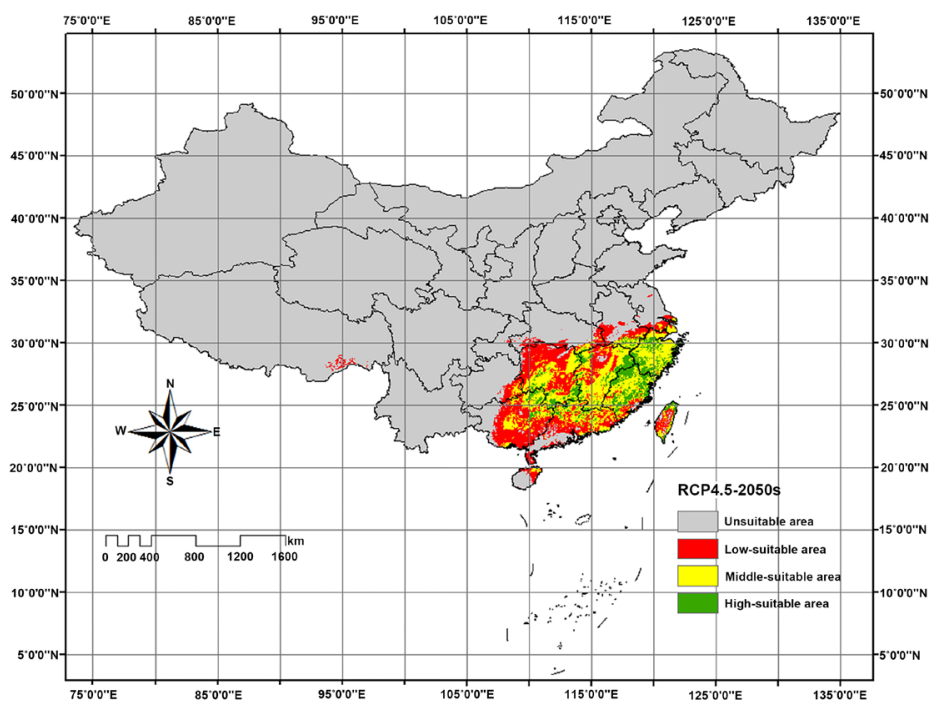

(a)

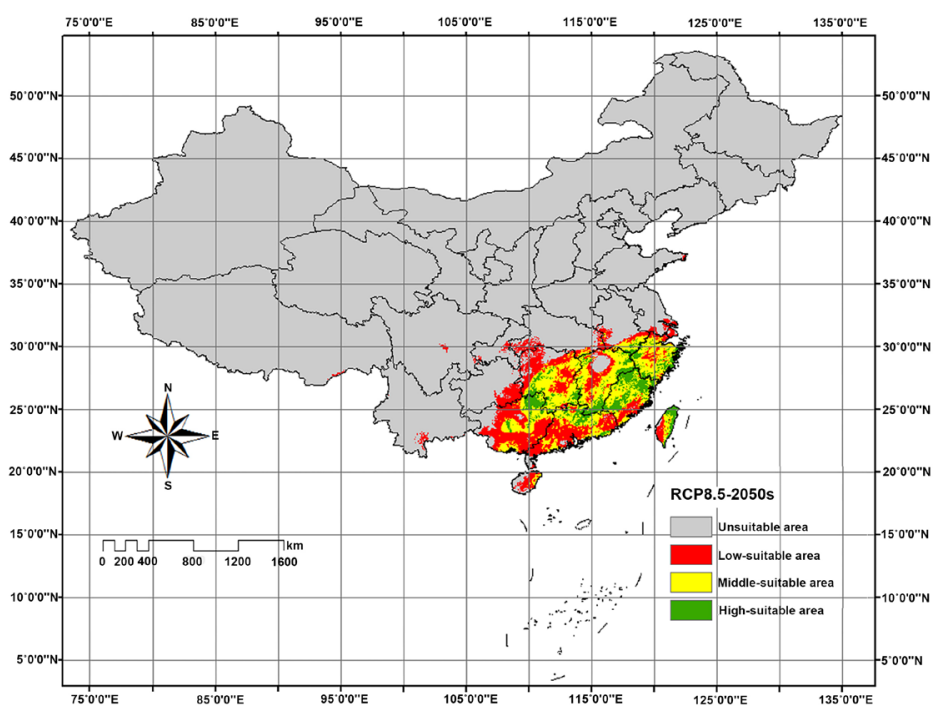

(b)

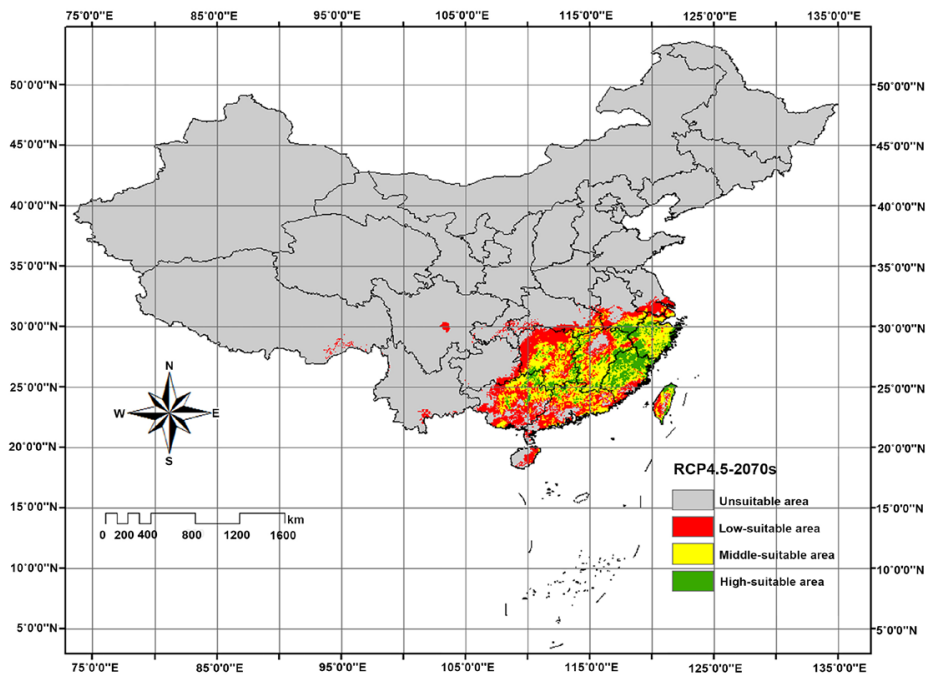

(c) 


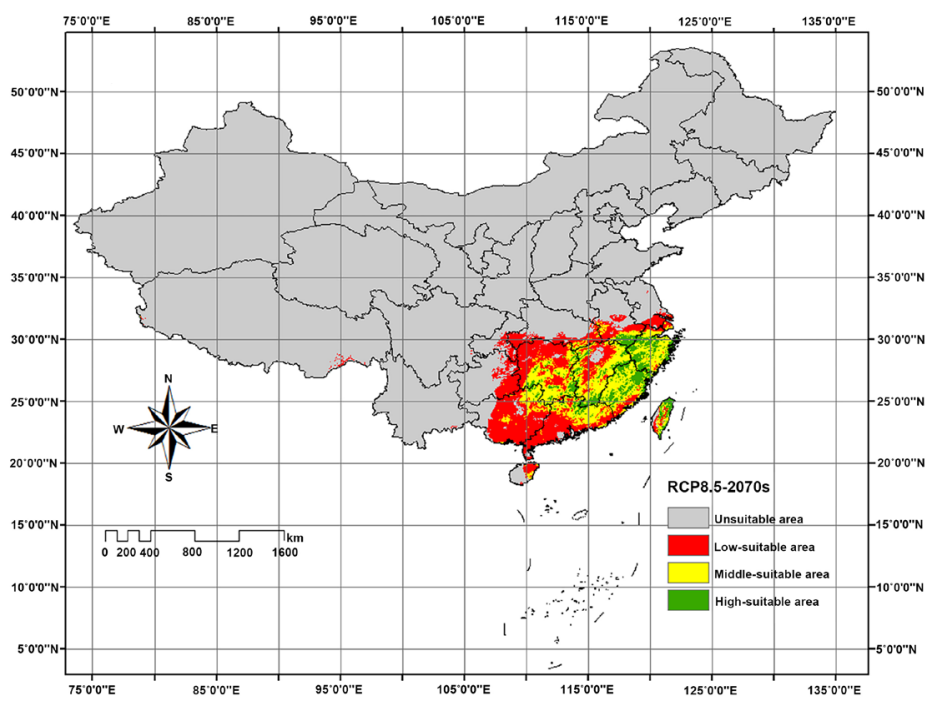

(d)

Figure 4. Potential distributions of Machilus thunbergii under future climatic conditions. (a) Distributions of 2050s under RCP4.5; (b) Distributions of 2050s under RCP8.5; (c) Distributions of 2070s under RCP4.5; (d) Distributions of 2050s under RCP8.5.

and high-suitability areas decreased by $0.7 \times 10^{4} \mathrm{~km}^{2}$, and $2.93 \times 10^{4} \mathrm{~km}^{2}$, respectively. In general, the area of the high-suitability increases first and then decreases, and the area of the medium-suitability area decreases more significantly under the RCP8.5.

\section{Discussion}

\subsection{Main Climatic Factors Affecting the Distribution of Machilus thunbergii}

Under the current climate, Machilus thunbergii is mainly distributed in southeastern China, roughly correspond with the geographical distribution of $\mathrm{Ma}$ chilus thunbergii with the previous research (Takyu \& Ohsawa, 1997; Utteridge, 2010; Kaneko et al., 2012).

According to the influence of environmental variables on the distribution of Machilus thunbergii, the Precipitation of coldest quarter (Bio19), the Precipitation of driest month (Bio14), the Annual precipitation (Bio12), the Mean diurnal range (Bio2), the Temperature annual range (Bio7), are the most important environmental factors affecting the distribution of Machilus thunbergii. It shows that Machilus thunbergii has high requirements for precipitation and temperature, and the influence of precipitation is greater than temperature. It shows that precipitation is the limiting factor determining the distribution of Machilus thunbergii. There is abundant rainfall in the eastern of subtropical China, which is coincident with the suitable area of Machilus thunbergii.

The flower bud differentiation period generally from August to September. It blooms in February, and enters fruit maturation period from June to July. The 
seeds can germinate in a suitable environment when they fall to the ground (Kaneko et al., 2012; Xu, 2014). Adverse meteorological during flowering, such as low temperature in winter and late frost in spring, can lead to severe freezing injury on flower buds. Low temperature or high temperature, drought, water logging and so on during the young fruit-growing period will cause a lot of fruit drop.

Some studies have pointed out that precipitation makes a difference on reproduction and physiology of Machilus thunbergii (Jiang, 2011). A high germination rate requires a moisture content of Machilus thunbergii seeds above 40\% (Lin \& Chen, 1995; Jiang et al., 2005). The annual precipitation can influence on the fruit size (Jiang, 2011), which is an important reason for the biennial bearing (Wang et al., 2010). Under natural conditions, precipitation is the decisive factor that directly affects the air humidity and water content of soil in the community environment. The air which is too dry and soil with low water content will become the limiting factors of photosynthesis (Tranquillini et al., 1979). Compared with the western plateau and the northern plain with greater environmental changes, the climate in the third ladder of China's topography (Zhejiang, Fujian, Jiangxi, Taiwan and other places) and the islands on the East China Sea, and the islands on the East China Sea is relatively mild. It can be inferred that the distribution of the suitable areas is related to the stable climate in the east of China. Indicating that precipitation, especially in winter, and stable temperature environment determine the geographical distribution of Machilus thunbergii by influencing its growth and renewal.

With global warming and increasing precipitation, the suitable area of $\mathrm{Ma}$ chilus thunbergii will expand in the future. However, the environmental factor response curve (Figure 2) shows that the distribution probability of Machilus thunbergii increases with the increase of environmental factors within a certain range, and decreases after the peak. Therefore, under the RCP4.5, the increased area between the 2050s and 2070s is not obvious. And under the RCP8.5, the low-suitability area continues to expand, while the medium-suitability and high-suitability areas tend to stabilize and even decline.

It indicates that, with the climate change, the suitable area of Machilus thunbergii will not continue to increase, and may even restrain its expansion after reaching a certain peak.

\subsection{Nonclimatic Factors Affecting the Distribution of Machilus thunbergii}

According to the simulation, the medium-suitability area and the high-suitability are mainly concentrated in Wuyi Mountains, Luoxiao Mountains, Xuefeng Mountains, Nanling and Taiwan Mountains, maintaining a relatively stable state. The distribution area is mainly hilly mountains with complex terrain (Dordel et al., 2011), where the microclimate can maintain a relatively stable habitat and reduce the impact of climate change on Machilus thunbergii.

In addition, the northward migration of the suitable area of Machilus thun- 
bergii may relate to vegetation type. It can be seen that, in the future, the distribution area of Machilus thunbergii moves northward, preferring areas with more deciduous trees. With the climate change, the interspecific and intraspecific competition may become more intense. And studies have shown that when the neighboring plants are deciduous broad-leaved species, the basal diameter growth and tree height of Machilus thunbergii is significantly greater (Massey et al., 2006; Tong et al., 2013), because deciduous broad-leaved humus can promote the growth of saplings better than evergreen leaved humus (Koorem et al., 2011).

Furthermore, the rate of spread of the communities may not keep pace with the rate of global climate change. Under the scenario of global climatic change, leading to warming, altered precipitation patterns and extreme weather events (drought, flood) will increase their impact on forest ecosystems (growth, migration and disappearance, species invasion, etc.) in the future, posing a serious threat to forest security (Yang, 2017). In addition, climate warming will cause some unexpected events, such as wildfire caused by extreme high temperature and drought, leading to the extinction of species, the rapid loss of forest and other unpredictable harm, and further aggravate the rate of climate change. Therefore, the migration and expansion of the suitable zone of Machilus thunbergii in the future may not proceed as the simulation results.

\subsection{Conservation and Development Strategies for Machilus thunbergii}

The research results can provide theoretical reference for the promotion and planting of Machilus thunbergii. The medium-suitability and high-suitability areas such as Zhejiang and Fujian can be given priority and emphasis for planting division and germplasm resources protection when making planting area plan, while the planting scale can be appropriately expanded in the low-suitability areas. Carrying out germplasm resource protection in the relatively stable distribution area and setting up protective forest belt in the periphery of the area is of significance to form a transition area for the development and expansion of natural Machilus thunbergii.

\section{Acknowledgements}

The authors thank the Cooperation Plan of Zhejiang Province and Chinese Academy of Forestry (grant number 2018SY08) for funding this study.

\section{Conflicts of Interest}

The authors declare no conflicts of interest regarding the publication of this paper.

\section{References}

Adhikari, D., Barik, S. K., \& Upadhaya, K. (2012). Habitat Distribution Modelling for Reintroduction of Ilex khasiana Purk., a Critically Endangered Tree Species of Nor- 
theastern India. Ecological Engineering, 40, 37-43.

https://doi.org/10.1016/j.ecoleng.2011.12.004

Chen, Y. R., Xie, H. M., Luo, H. L., Yang, B. Y., \& Xiong, D. J. (2019). Impacts of Climate Change on the Distribution of Cymbidium kanran and the Simulation of Distribution Pattern. Chinese Journal of Applied Ecology, 30, 3419-3425.

Dordel, J., Seely, B., \& Simard, S. W. (2011). Relationships between Simulated Water Stress and Mortality and Growth Rates in Underplanted Toona ciliata Roem. in Subtropical Argentinean Plantations. Ecological Modelling, 222, 3226-3235.

https://doi.org/10.1016/j.ecolmodel.2011.05.027

Elith, J., Phillips, S. J., Hastie, T., Dudík, M., Chee, Y. E., \& Yates, C. J. (2011). A Statistical Explanation of MaxEnt for Ecologists. Diversity and Distributions, 17, 43-57. https://doi.org/10.1111/j.1472-4642.2010.00725.x

Fandohan, A. B., Oduor, A. M. O., Sodé, A. I., Wu, L., Cuni-sanchez, A., Assédé, E., \& Gouwakinnou, G. N. (2015). Modeling Vulnerability of Protected Areas to Invasion by Chromolaena odorata under Current and Future Climates. Ecosystem Health and Sustainability, 1, 1-12. https://doi.org/10.1890/EHS15-0003.1

FAO (2010). Global Forest Resources Assessment 2010. Rome: Food and Agriculture Organization of the United Nations.

FAO (2015). Country Report: Brazil. Rome: Global Forest Resources Assessment.

Fick, S. E., \& Hijmans, R. J. (2017). WorldClim 2: New 1-km Spatial Resolution Climate Surfaces for Global Land Areas. International Journal of Climatology, 37, 4302-4315. https://doi.org/10.1002/joc.5086

Hu, S. Q. (2002). New Landscape: Machilus thunbergii. Zhejiang Forestry, 12, 29.

Hwang, M. Y., Lin, K. H., Yang, M. M., Chou, H. M., Yang, C. M., \& Chang, Y. T. (2011). Chlorophyll Fluorescence, Spectral Properties, and Pigment Composition of Galls on Leaves of Machilus thunbergii. International Journal of Plant Sciences, 172, 323-329. https://doi.org/10.1086/658157

Jiang, R. B. (2011). Genetic Diversity and Good Provenance Selection of Machilus thunbergii. Beijing: China Academy of Forestry Sciences.

Jiang, X. M., \& Yu, X. (2001). An Introduction to and the Research Progress on Machilus thunbergii. Acta Agriculture Universitatis Jiangxiensis, 23, 231-235.

Jiang, X. M., Wu, S., Wan, N. N., Yang, G. H., Gong, B., \& Ye, J. S. (2005). Phenology Observation and Seed-Collecting and Seedling-Nursing Techniques of Machilus thunbergii. Jiangxi Forestry Science and Technology, 5, 1-4.

Kaneko, Y., Lian, C., Watanabe, S., Shimatani, K. I., Sakio, H., \& Naohiko, N. (2012). Development of Microsatellites in Machilus thunbergii (Lauraceae), a Warm-Temperate Coastal Tree Species in Japan. American Journal of Botany, 99, 265-267. https://doi.org/10.3732/ajb.1100560

Koorem, K., Price, J. N., \& Moora, M. (2011). Species-Specific Effects of Woody Litter on Seedling Emergence and Growth of Herbaceous Plants. PLoS ONE, 6, e26505. https://doi.org/10.1371/journal.pone.0026505

Kramer-Schadt, S., Niedballa, J., Pilgrim, J. D., Schröder, B., Lindenborn, J., Reinfelder, V. et al. (2013). The Importance of Correcting for Sampling Bias in MaxEnt Species Distribution Models. Diversity and Distributions, 19, 1366-1379. https://doi.org/10.1111/ddi.12096

Le Quéré, C., Andrew, R. M., Friedlingstein, P., Sitch, S., Pongratz, J., Manning, A. C. et al. (2018). Global Carbon Budget 2017. Earth System Science Data, 10, 405-448. https://doi.org/10.5194/essd-10-405-2018 
Li, X., Li, Y., \& Fang, Y. M. (2018). Prediction of Potential Suitable Distribution Areas of Quercus fabri in China Based on an Optimized Maxent Model. Scientia Silvae Sinicae, 54, 153-164.

Lin, T. P., \& Chen, M. H. (1995). Biochemical Characteristics Associated with the Development of the Desiccation-Sensitive Seeds of Machilus thunbergii Sieb. \& Zucc. Annals of Botany, 76, 381-387. https://doi.org/10.1006/anbo.1995.1111

Ma, L. Y., \& Huang, J. J. (2019). The Research Progress and Application Prospect of Native Tree Species Machilus thunbergii. Protection Forest Science and Technology, 6, 66-68.

Massey, F. P., Massey, K., Press, M. C., \& Hartley, S. E. (2006). Neighbourhood Composition Determines Growth, Architecture and Herbivory in Tropical Rain Forest Tree Seedlings. Journal of Ecology, 94, 646-655. https://doi.org/10.1111/j.1365-2745.2006.01127.x

SFA (2014). China Forest Resources Report (2009-2013). Beijing: State Forestry Administration of China, China Forestry Press.

Sung, S., Kwon, Y. S., Lee, D. K., \& Cho, Y. (2018). Predicting the Potential Distribution of an Invasive Species, Solenopsis invicta Buren (Hymenoptera: Formicidae), under Climate Change Using Species Distribution Models. Entomological Research, 48, 505-513. https://doi.org/10.1111/1748-5967.12325

Takyu, M., \& Ohsawa, M. (1997). Distribution and Regeneration Strategies of Major Canopy Dominants in Species-Rich Subtropical/Warm Temperate Rainforests in South-Western Japan. Ecological Research, 12, 139-151. https://doi.org/10.1007/BF02523779

Tian, Z. P., \& Jiang, D. B. (2013). Climate Simulation Capability Analysis of East Asia and China with Different Resolution CCSM4. Chinese Journal of Atmospheric Sciences, 37, 171-186.

Tong, Y. W., Xiang, W. H., Wang, Z. W., Durka, W., \& Fischer, M. (2013). Effects of Topography, Neighboring Plants and Size-Dependence of Machillus thunbergii on Sapling Growth and Survivorship. Biodiversity Science, 21, 269-277. https://doi.org/10.3724/SP.J.1003.2013.09008

Tranquillini, W. (1979). Physiological Ecology of the Alpine Timberline: Tree Existence at High Altitude with Special Reference to the European Alps, Ecological Studies. New York: Springer Berlin Heidelberg. https://doi.org/10.1007/978-3-642-67107-4

Utteridge, T. M. A. (2010). Menispermaceae through Capparaceae by Flora of China Editorial Committee. Flora of China, 65, 351.

Wang, S. P., Xie, Z. H., Wang, G. P., \& Liu, L. (2010). Seedling Cultivation Technology of Machillus thunbergii. Modern Agricultural Science and Technology, 5, 194-195.

Wu, S. H., Hwang, C. Y., Lin, T. P., Chung, J. D., Cheng, Y. P., \& Hwang, S. Y. (2006). Contrasting Phylogeographical Patterns of Two Closely Related Species, Machilus thunbergii and Machilus kusanoi (Lauraceae), in Taiwan. Journal of Biogeography, 33, 936-947. https://doi.org/10.1111/j.1365-2699.2006.01431.x

Wu, Z. Y., Raven, P. H., \& Hong, D.Y. (2008). Flora of China, Vol. 7 (Menispermaceae through Capparaceae). Beijing and St. Louis: Science Press and Missouri Botanical Garden Press.

Xu, D. (2014). The Population Structure of Machilus thunbergii and Effect of Animals on Its Seed Dispersal. Master Thesis, Nanjing: Nanjing Forestry University.

Yang, X. Q., Kushwaha, S. P. S., Saran, S., Xu, J., \& Roy, P. S. (2013). Maxent Modeling for Predicting the Potential Distribution of Medicinal Plant, Justicia adhatoda L. in Lesser Himalayan Foothills. Ecological Engineering, 51, 83-87. 
https://doi.org/10.1016/j.ecoleng.2012.12.004

Yang, Y. S. (2017). The Impacts of Global Environmental Changes on Typical Ecosystems: Status, Challenges and Trends. Acta Ecologica Sinica, 37, 1-11. https://doi.org/10.5846/stxb201701050044

Zhang, G. S. (2010). Study on the Intraspecific and Interspecific Competition in Machilus thunbergii Community. Journal of Fujian College of Forestry, 2, 20.

Zhang, L., Liu, S., Sun, P., Wang, T., Wang, G., Zhang, X., \& Wang, L. (2015). Consensus Forecasting of Species Distributions: The Effects of Niche Model Performance and Niche Properties. PLOS ONE, 10, e0120056.

https://doi.org/10.1371/journal.pone.0120056

Zhang, Z. M., Yang, G. D., Xie, M. M., Zhong, Y. Q., Zhai, F.F., Wang, X. R., \& Yi, X. G. (2019). Community Structure and Environmental Interpretation of Machilus thunbergii in Mountain Longchi, Jiangsu Province. Chinese Journal of Ecology, 38, 1637-1645. 